\title{
Intelligent Supervisory Control of Discrete Event Systems - A Holon based Approach
}

\author{
Oliver L. Iliev ${ }^{1}$, Kire Jakimovski ${ }^{1}$, and Ahmad Zakeri ${ }^{2}$ \\ ${ }^{1}$ FON University, FICT, Bul. Vojvodina bb, \\ 1000 Skopje, Macedonia \\ \{oliver.iliev, kire.jakimovski\}@ fon.edu.mk \\ ${ }^{2}$ University of Wolverhampton, School of Engineering and the Built Environment, Wulfruna \\ Street, Wolverhampton WV1 1SB, United Kingdom \\ A.Zakeri2@wlv.ac.uk
}

\begin{abstract}
Supervisory Control of Discrete Event Systems (DEVS) requires potential capabilities for constant adaptation and fast responses to the frequent unexpected disturbances occur in process and changes in production orders. Both hierarchical and heterarchical architecture control systems being developed so far have had limited success in achieving such potential capabilities. Hierarchical architecture systems due to their rigid structures inhibit inadequate responses to the process disturbances. Heterarchical architecture systems are quite good in dealing with the disturbances and are highly adaptable, but such structures cannot guarantee high performance due to unpredictable behavior. The shortcomings of the said systems develops the need for designing Holon based structure in which entities are able to communicate, cooperate, and have certain level of intelligence that enable them to be highly autonomous. In this paper we present a methodology that encapsulates individual needs of the physical layer, in general discrete event objects, in order to promote a goal orientated infrastructure with negotiation and cooperation capabilities at the computational layer of Discrete Event Systems. This methodology propagates chains of cooperation between holons seeking satisfactory goal realization. Architecture for a Holonic-based intelligent Supervisory Control of DEVS has been proposed along with a guideline for its product ionisation. The newly proposed taxonomy of individual holons occurring in a discrete event system as a supervisory controller will allow to understand interaction mechanisms in a complex Holon's society, and to obtain design methodology for a Holonic based control of DEVS.
\end{abstract}

Keywords: Intelligent Control, Discrete Event Systems (DEVS), Holon, Holarchy, Holonic Manufacturing Systems (HMS), Intelligent Manufacturing Systems (IMS) 


\section{Introduction}

The manufacturing industry has been facing a continuous change from a supplier's to a customer's market. The growing industrial capacity provides the customer wider choice, which in return increase suppliers competition. As a result, the customers are becoming more and more demanding and less loyal. The company survival must lie in constant product innovation, low-cost product customization, improved customer service, and delivering the product which best meets customer requirements. Due to globalization, these trends will even increase in the future [14]. It is very difficult to estimate what will be the business requirements in the 21 st century, but the current requirements of producing goods of a specific quality at low costs will certainly remain. Beside this, the companies must shorten product-life cycles, reduce time-to-market, increase product variety, instantly satisfy demand, while maintaining quality and reducing investment costs. As a summary, the companies must to:

- Increase products complexity

- $\quad$ Fasten the process of changing products

- Fasten the process of introduction products

- Decrease the investments per product

It is obvious that this structural reengineering will lead to the increasing of system complexity and continual change under decreased costs.

\section{New conditions in $21^{\text {st }}$ century manufacturing}

The upcoming challenges of the 21st century manufacturing include improving the Intelligent Control of Flexible manufacturing systems (FMS) in terms of incorporating new complex organizational and functional capabilities. Due to the time constraints a building-block or "plug and play" capabilities for developing and operating a manufacturing system should be applied. Yet the slow and limited industrial take-up indicates that it is still difficult to convince potential users of the benefits of this approach [1,2]. Most existing requirements placed on a manufacturing operation will still apply in the future. These include guaranteed performance, high reliability of equipment, quality assurance, cost minimization etc. Given the trends, additional requirements will become relevant, if not predominant.

One of the major requirements will be to minimize the complexity of the manufacturing process (despite the likely increases in the variety of products and product ranges). This can be achieved by reducing the number of manufacturing system components and by standardizing structure of these components and their interaction. Nevertheless, there is a limit to reduction and standardization, as a complex product requires a certain set of complex operations. At the same time, the remaining process complexity must be mastered. This can be achieved on the one hand by creating an intuitive, self-explaining structure of the control system, and on the other hand by assuring a well-defined behavior upon certain actions and events. Ideally, the control layer of a manufacturing system should be completely transparent to the end-user, and any actions or events should exhibit well-known effects on the overall system performance. In particular, the control layer should not introduce additional complexity 
and the overall behavior of a manufacturing system should be well-defined under all circumstances. Constant product changes require the re-use of existing manufacturing equipment.

Re-use of manufacturing units can be achieved either through flexibility of function or through re-configurability $[10,11]$. A unit is immediately re-usable if the new operations required are part of the range and mix of operations of this unit. High functional flexibility thus increases the chances of equipment re-use. Units equipped (up front) with a large range of operations, however, can be very costly. In contrast, the costs of a unit are often reduced considerably if the re-use is provided through manual re-configurability. For monthly product changes, this is acceptable. Weekly or daily product changes, though, are likely to require instant unit flexibility. At the same time, this requirement applies also to the process reorganization. The manufacturing process must be either flexible or reconfigurable in order to deal with the product changes. In the former case, the manufacturing system is sufficiently flexible to change to the new processing steps. In the latter, the manufacturing system itself has to be re-organized in order to create the desired processing steps (including rearrangement of units and rerouting of parts).

From the market point of view, the volatility of the demand forces the vendors to adapt their output. Failing in supply the right product at the right time jeopardize the market share. Also, the manufacturing system must be able to vary its production output. This implies scalability of the manufacturing system if the total volume changes, and inter-product flexibility if the product mix changes. Scalability can be achieved either by extending the working time or by adding more resources. Extending the working time is certainly limited to 24 hours a day and seven days a week. The task of managing change becomes even more difficult if it has to be achieved at decreasing costs. A low investment approach to change management, however, creates a difficulty of disturbances. The system robustness can be achieved either structurally or dynamically. Buffers in terms of material or time slack provide structural robustness.

As a result of all previously mentioned, these requirements will have serious implications for the control of such a system. Many requirements can only be achieved if the control system meets equivalent requirements. Requirements like unit flexibility or re-configurability are mainly hardware issues, but system responsiveness is certainly impossible without high level of intelligent control of this class of discrete event systems.

- The architecture of the control should be decentralized and product-/resourcebased. For even small manufacturing systems, a centralized approach to control is practically impossible. A single controller would be too complex, would become a bottleneck, and would be too difficult to change. There must be at least some kind of decentralization.

- Control interactions should be abstract, generalized and flexible. A resource-based control system is certainly easier to change and scale up than a centralized or functionally decentralized system. Maximum changeability can be achieved only if dependencies between resources are reduced to a minimum. Consequently, in order to achieve maximum changeability, resources should be de-coupled in these steps; abstract interaction; generalized interaction and flexible acquaintances and interaction 
- The control should be reactive and pro-active. To respond to short-term changes and disturbances, the control must be reactive. This includes the ability to recognize critical situations, make decisions about the reaction, and perform corresponding actions.

- The control should be self-organizing. The need to adapt the manufacturing process in the face of changes or disturbances will not only affect the resources, but also the organization of the manufacturing process as a whole. In a highly responsive manufacturing system, the organization must be responsive too and this responsiveness should emerge from any (re-)configuration of the resources and rearrangement of the process.

\section{The Holon Concept}

The main idea of the Holon model stems from the work of Arthur Koestler [16]. He postulated a set of underlying principles to explain the self-organizing tendencies of social and biological systems. Proposed the term Holon describe the elements of these systems as combination of the Greek word holos, meaning "whole", with the suffix -on meaning "part". This term reflects the tendencies of holons to act as autonomous entities, yet cooperating to form apparently self-organizing hierarchies of subsystems, such as the cell/tissue/organ/system hierarchy in biology.

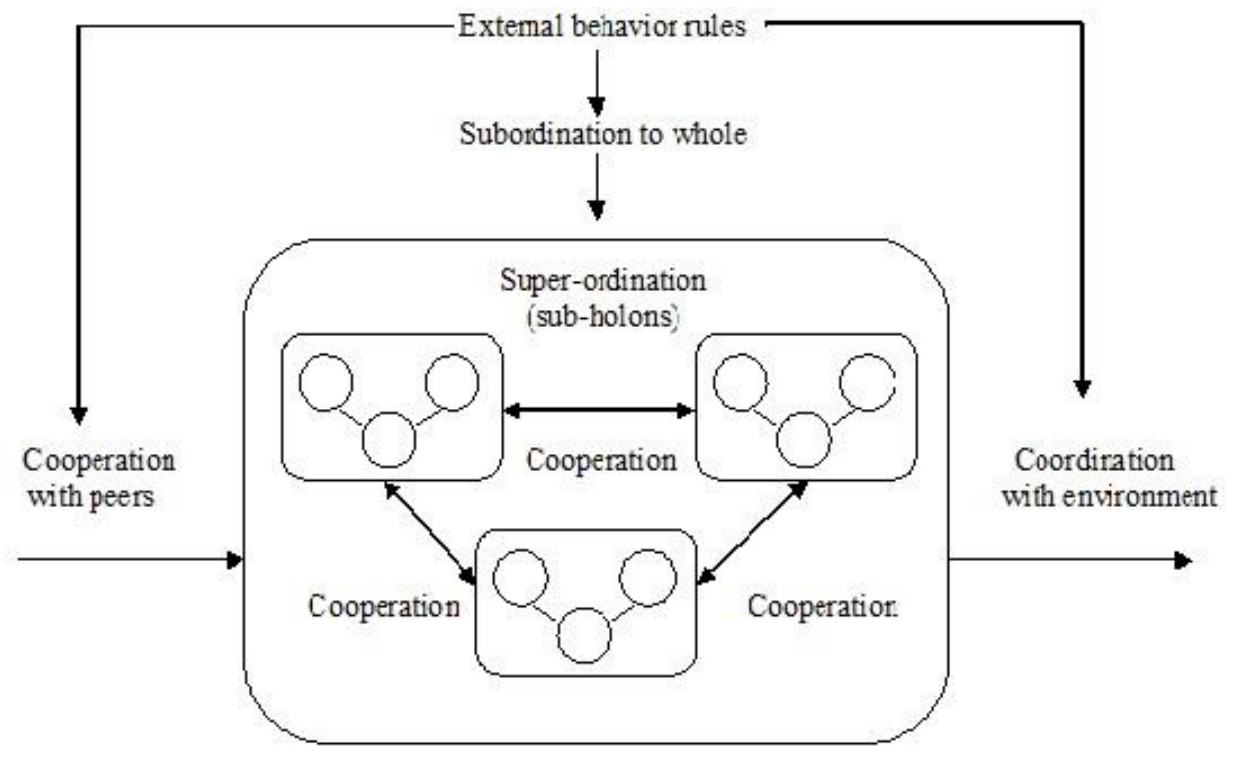

Fig.1. Generic model of a Holarchy

Koestler has identified structural patterns of self-replicating structures, named holarchies, as a universal principle of self-replicating structures of nested hierarchies intrinsically embedded in the functionality of natural systems. Holarchies have been 
envisioned as models for the Universe's self-organizing structure in which holons at several levels of resolution in the nested hierarchy [16] Proposed structure (Fig.1) behave as autonomous wholes and yet as cooperative parts for achieving the goal of the Holarchy. In such a nested hierarchy each Holon is a sub-system retaining the characteristic attributes of the whole system. What actually defines a Holarchy is a purpose around which holons are clustered and subdivided in sub-holons at several levels of resolution according to the organizational dissectibility required [17].

From a software engineering perspective a Holon, as a unit of composition retaining characteristic attributes of the whole Holarchy can be viewed as a class [15, 22]. Thus the object-oriented paradigm seemed suitable for modeling holarchies as software systems. Within a Holarchy, holons can belong to different clusters simultaneously, displaying rule governed behavior. In this context, holons are essential in hierarchical systems with intelligent performance. They allow the modeling of complex phenomena in a non-reductionist way. In a multi-strata hierarchy, in the sense of M. Mesarovic [18], holons are the components for modeling parts of the system at different levels. They emerge in this case from the dependent holons in the model of the next lower level. This concept allow to obtain a rigorous mathematical approach to construct models with this level of complexity. A different situation is given by hierarchies which are multi-layer systems, where the components receive "orders" from components above and transmit "orders" to components on the next lower layer of the model. When Arthur Koestler introduced his concept of a "Self-organizing Open Hierarchical Order" (SOHO) he had a multi-layer hierarchy in mind with holons as the components.

Systems Theory provides formal models for solving complex problems in science and engineering, has the task to elaborate the concept of Holon and related Holonic models and to provide methods and computerized tools for its application [21]. However, this conceptual framework has a much wider domain of application, for example any living organism, a forest but also cells or a fully automated manufacturing system, are real systems to which this framework can be applied. Hierarchies are already models in a decomposed form. The different control- and communication channels between the holons constitute the coupling system of the decomposition. A hierarchy with holons as its components, a Holarchy, constitutes a very desirable decomposition of the overall system. Then "intelligent" behavior of the level components are required and selforganization of some kind might be necessary to meet the performance requirements [5].

When looking "down", a Holon represents a quasi-autonomous whole (self-assertion tendency) such that the depending holons of the next level have for performing their main function no need in coupling their input- and output channels to other holons. On the other hand, looking "up", a Holon integrates its functions into an existing or developing whole which can be defined as an integration ability. Hierarchical functioning of a Holarchy distinguishes between input and output hierarchies.

Input hierarchies operate to achieve from the signals and states associated with holons on lower levels an abstraction or generalization represented by the signals and states of holons on upper levels. Therefore their main function is to compute the emergent properties in a Holarchy.

Output-hierarchies, on the other hand, are defined as holarchies which operate in the opposite direction. They take signals and states from holons of upper levels and transform them to specific concrete signals and states suitable for the proper operation in holons of the lower levels of a Holarchy. The holons of a SOHO-structure have to be 
balanced between being "mechanized" and having a certain degree of "freedom". Holons on higher levels have usually more freedom for their operation while holons at lower levels will usually have to follow more mechanized patterns in their operation. Dynamical equilibrium of these tendencies is achieved through self-assertion tendency and the integration tendencies of the holons counterbalance each other. Disorder appears if those tendencies dominate each other. Also, a very important property of a SOHOstructure is regeneration. Critical challenges caused by the environment of a Holarchy may result in changes of rules for operating holons such that an adaption to new circumstances is realized by a reached new state of equilibrium.

Koestler states that the rules of a social Holon are not reducible to the rules which conduct its members. He writes in Definition 9.8 of his SOHO-structure "The egotism of the social Holon feeds on the altruism of its members".

\section{Holon based control of DEVS}

Regarding the DEVS control based on holons approach, there are two key observations:

- These systems evolve and grow to satisfy increasingly complex and changing needs by creating stable "intermediate" forms which are self-reliant and more capable than the initial systems.

- In living and organizational systems it is generally difficult to distinguish between 'wholes' and 'parts': almost every distinguishable element is simultaneously a whole (an essentially autonomous body) and a part (an integrated section of a larger, more capable body).

Suda's observation $[23,24]$ was that such properties would be highly desirable in a manufacturing operation which was subject to increasingly stringent demands and faster changes. He therefore proposed a building block or "Holon" based model for designing and operating elements comprising manufacturing processes. Some key properties of a Holonic based control system are:

- Autonomy: The capability of a manufacturing unit to create and control the execution of its own plans and/or strategies (and to maintain its own functions).

- Cooperation: The process whereby a set of manufacturing units develop mutually acceptable plans and execute them.

- Self-Organization: The ability of manufacturing units to collect and arrange themselves in order to achieve a production goal.

- Reconfigurability: The ability of a function of a manufacturing unit to be simply altered in a timely and cost effective manner.

- Openness: The system must be able to accommodate the incorporation of new holons, the removal of existing holons, or modification of the functional capabilities of existing holons, with minimal human intervention, where holons or their functions may be supplied by a variety of diverse sources.

In this context, a Holon is "an autonomous and cooperative building block of a manufacturing system for transforming, transporting, storing and/or validating information and physical objects" [8]. It consists of a control part and an optional physical process-sing part. A Holon can itself consist of other holons which provide the 
necessary processing, information, and human interfaces to the outside world. From this point of view a Holarchy can be defined as a "system of holons which can co-operate to achieve a goal or objective". Holarchies are created and dissolved dynamically. Intelligent supervisory control system for a DEVS must provide a framework for the unambiguous specification of the structure and relationship among functional units in the system (Fig.2).

A manufacturing Holon is an autonomous and cooperative building block of a manufacturing system for transforming, transporting, storing and/or validating information, and/or physical objects. Therefore a manufacturing holons usually comprises knowledge and software components with a hardware component as an option. Functionally, a holons may be considered to comprise an intelligent control system and a processing system.

Intelligent Control system is responsible for execution of the control plan for the process being controlled. The control block may include besides traditional control algorithms rule-based reasoning, fuzzy logic, and neural nets. As a part of this system is inter holons interface which handle the inter-holons communication, negotiation and cooperation. Intelligent control systems consist of control/regulation components, and process-sing subsystem. The processing system consists of all processing components necessary to realize a manufacturing activity as transforming, transporting, storing and/or validating information and/or physical objects. This system is responsible for the interacting behavior of the internal components, as well as the set of procedural rules and decision-making functions that govern the interaction of the components. On the other hand, inter holons interface is responsible for Holon's communication and operation, e.g. provide the context where holons may locate, contact and interact with each other. Actual realization of this structure allow to be incorporated in the ICS and that all cooperation domains may be dynamically generated by the operations of holons' constituent parts $[12,13]$.

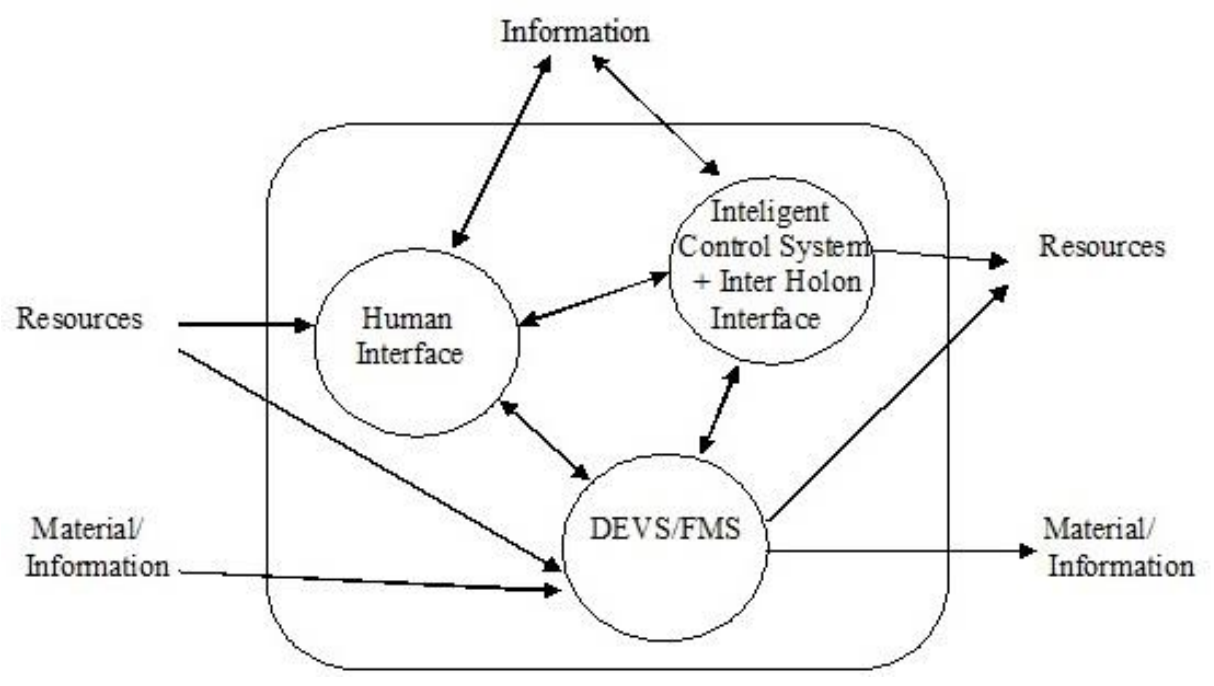

Fig.2. Generic Model of Holon based Control System for DEVS 
Inter Holon interface comprises the following elements:

- Facilities transient messages passing between holons and the cooperation domain.

- Decision making mechanisms to support holons in their activities, such as task planning, negotiation and information exchange.

- Techniques and rules to decompose and allocate tasks among compound holons, as well as facilities to schedule and control tasks within a Holon.

- Facilities to monitor the status of a distributed task, and schedule/control all actions within this task.

$D E V S / F M S$ provides the logical and physical interface to the DEVS/FMS system through a suitable communication network [25]. Real-time communication is supported through a Real-time layer. The DEVS/FMS system may itself contain intelligent elements such as e.g. self-diagnosis, process model-oriented diagnosis, etc.

The human interface comprises the interfaces to humans such as operators, supervisors, maintenance personnel, and process engineers. It may include front ends, diagnostic and explaining components. A Holon may be composed of a set of other holons in a recursive containment Holarchy to form a compound or parent Holon. In this case, lower level holons included within the Holon cooperate with each other through their respective cooperation domains to generate task plans and to carry out these tasks $[26,27]$. In the case where a Holon does not include lower level holons, the internal cooperation domain represents the Holon's private autonomous functions and information.

Building of Holonic control system goes through development of certain Holon's interactions. In the beginning, a Holonic manufacturing system only consists of a set of unorganized resource holons which form a manufacturing Holon. Upon arrival of an order, however, the manufacturing Holon creates an order Holon which starts to negotiate with resource holons on the provision of certain manufacturing operations. During the negotiation process, the order Holon demands specific properties of the operation, such as high quality or high throughput, while the resource holons try to maximize their utilization. At the end of the negotiation, the resource holons move to form the agreed manufacturing line and the order Holon initiates the creation of work piece holons. At the next stage, the work piece holons enter the manufacturing Holarchy and immediately bargain for resources in order to get processed. Each work piece Holon does so individually and focuses on the next operation. After the performance of the operations, the work piece reinitiates the bargaining with the remaining operations. The overall organization of the resource Holarchy - initially or subsequently negotiated between order and resource holons - assures that the work piece load is efficiently distributed over the available resources in order to achieve the global goals of this Holarchy [19, 20].

In case of a disturbance, the affected resource Holon removes itself from the resource Holarchy and goes to a repair booth. The remaining resource holons re-organize themselves in order to account for the capacity loss. From the work piece holons point of view, the processing continues as usually, only with less resource holons to bargain with. After repair, the resource Holon tries to join the resource Holarchy again. At the end of the order processing, the order Holon is removed and the resource Holarchy dissolves into the resource holons which then try to participate in new order holarchies. 
Intelligent control of DEVS must satisfy the requirements demanded by that Holon's autonomy, cooperation and openness roles. Contrary to traditional manufacturing paradigms, holons are managed in a distributed fashion through interaction with their respective Holonic structure. This structure assists the holons by offering services including:

- Selecting appropriate function blocks to provide the autonomous skills needed to perform a given task.

- Managing data/event flows between function blocks.

- $\quad$ Supporting interaction and negotiation protocols etc. with other holons through inter Holon interface.

- $\quad$ Access to data/knowledge bases via suitable interfaces.

- Assisting in task decomposition, information filtering, creating and validating schedules, and handling interrupts.

\section{Conclusion}

In this paper, we propose architecture for a Holon based intelligent Supervisory Control of DEVS. The proposed taxonomy of individual holons occurring in a discrete event system as a supervisory controller allow to understand interaction mechanisms in a complex Holon's society, and to obtain design methodology for a Holonic based control of DEVS. Modern flexible manufacturing systems require potential capabilities for constant adaptation and fast responses to the frequent unexpected disturbances occur in process and changes in production orders. So far, developed control architectures have had limited success in achieving such potential capabilities. Limitations of these system should be overcome with designing of Holon based structure in which entities are able to communicate and cooperate, and have certain level of intelligence that enable them to be highly autonomous. This methodology encapsulates individual needs of the physical layer, in general discrete event objects, in order to promote a goal orientated infrastructure with negotiation and cooperation capabilities at the computational layer of Discrete Event Systems. This methodology propagates chains of cooperation between holons seeking satisfactory goal realization.

\section{References}

1. Agre, J., Elsley, G., McFarlane, D., Cheng, J., Gunn, B.: Holonic Control of Cooling Control System. In Proceedings of the 4th Rensselaer International Conference on Computer Integrated Manufacturing and Automation Technology, Rensselaer, New York, NY, USA. (1994)

2. Amos, H., Ng, R., Yeung, W., Cheung, E.: HSCS - the design of a holonic shop floor control system. In Proceedings of the IEEE Symposium on Emerging technologies and Factory Automation, EFTA, Vol. 1, 179 - 185. (1996)

3. Van Brussel, H., Wyns, J., Valckenaers, P., Bongaerts, L. and Peeters, P.: Reference architecture for holonic manufacturing systems: PROSA. Computers in industry, Vol.37, No.3, 255-274. (1998) 
4. Christensen, J.: Holonic Manufacturing Systems - Initial Architecture and Standards Directi-ons. In Proceedings of the 1st European Conference on Holonic Manufacturing Systems, Hannover, Germany. (1994)

5. Deen, S.M., Johnson, C.A.: A Theoretical Foundation for Cooperating Knowledge Based Systems. In Proceedings of $11^{\text {th }}$ International Symposium on Methodologies for Intelligent Systems. (1999)

6. Gou, L., Hasegawa, T., Luh, P., Tamura, S., Oblak, J.: Holonic Planning and Scheduling for a Robotic Assembly Testbed. In Proceedings of the 4th Rensselaer International Conference on Computer Integrated Manufacturing and Automation Technology, Rensselaer, New York, USA. (1994)

7. Heikkilä, T., Jarviluoma, M., Hasemann, J.M.: Holonic control of a manufacturing robot cell," In Proceedings of the International Conference on Flexible Automation and Intelligent Manufacturing, Stuttgart, Germany. (1995)

8. Höpf, M.: Holonic Manufacturing Systems: The basic concept and report of IMS Test Case 5. NCMSInc. (1995)

9. Hayashi, H.: The IMS International Collaborative Program. 24th ISIR - Japan Industrial Robot Association, Japan. (1993)

10. Iliev, O.L., Dimirovski, G.M., Gough, N.E., Henry, R.M., Percinkova, B.R.: Computer -aided graph-network path-motion recognition in robotized MIMO FMS structures. In Proceedings of the B.Zajc, F.Solina (Eds.) IEEE Mediteranean Electrotechnical Conference (Selected Papers), Ljubljana, vol. II, 904-907. (1991)

11. Iliev, O.L., A.Zakeri, G.M.Dimirovski and N.E.Gough (1993), "Methodology for modelling and simulation of flexible manufacturing systems using stochastic Petri nets". In P.J.Nolan (Ed.) Proc.of Irish Manufact. Comm. Conf. on Innovation and Product Development, The IMC \& University College Galway, Galway (Eire), pp.1049-1061.

12. Iliev, O.L., Dimirovski, G.M., Gacovski, Z.M., Gough, N.E., Griffith, I.: Discrete event object oriented modelling of intelligent communication protocols. In World Automation Congress Symp on Intelligent Automation and Control, M.Jamshidi (Ed.), Vol. 4 of TSI Press Series on Robotics and Manufacturing (Selected Papers), Albuquerque NM (USA). (1996)

13. Iliev, O.L., Dimirovski, G.M., Gough, N.E.: Discrete event object-oriented modelling of intelligent communication protocols In Proceedings of the $11^{\text {th }}$ International Conference on System Engineering, Las Vegas, USA, 733-738. (1996)

14. Iliev O.L., Percinkova, B., Gough, N.E., Zakeri, A.: Two Level Control System for FMS - An Object Oriented Approach. In Proceedings of the 14th World Congress of International Federation of Automatic Control, Beijing , P.R. China. (1999)

15. Jennings, N.R.: Controlling Cooperative Problem Solving in Industrial Multi-Agent Systems using Joint Intentions. Artificial Intelligence, Vol.74, No.2. (1995)

16. Koestler, A.: The Ghost in the Machine. Hutchinson \& Co, London. (1967)

17. van Leeuwen, E., Norrie, D.: Holons and Holarchies. Manufacturing Engineer, Vol.76. (1997)

18. Mesarovic, M.D., Macko, D., Takahara, Y.: Theory of Hierarchical Multilevel Systems. Academic Press Inc., New York. (1970)

19. McFarlane, D.C.: Holonic Manufacturing Systems In Continuous Processing: Concepts And Control Requirements. In Proceedings od the Advanced Summer 
Institute (ASI) on Life Cycle Approaches to Production Systems, Lisbon, Portugal. (1995)

20. McFarlane, D.: Modular Distributed Manufacturing Systems and The Implications For Inte-grated Control. In the IEE Colloquium On Choosing The Right Control Structure, UK. (1998)

21. Simon, H.A.: The Architecture of Complexity. In Proceedings of the Amer. Phil. Society, Vol.106, No.6. (1962)

22. Shen, W., Norrie, D.H.: Agent-Based Systems for Intelligent Manufacturing: State of the Art Survey. Knowledge and Information Systems, Vol.1, No.2. (1999)

23. Suda, H.,: Future factory system formulated in Japan. Techno Japan, Vol.22, No.10, 15-25. (1989)

24. Suda, H.,: Future Factory System Formulated in Japan (2). Techno Japan, Vol.23. (1990)

25. Tanaya, P., Detand, J., Kruth, J.P.: Holonic Machine Controller: A study and implemen-tation of holonic behaviour to current NC controller. Computers in Industry, vol. 33. (1997)

26. Van Brussel, H.: Holonic Manufacturing Systems The Vision Matching the Problem. In Proceedings of the 1st European Conference on Holonic Manufacturing Systems, Hannover, Germany. (1994)

27. Van Brussel, H., Valckenaers, P., Bongaerts, L., Wyns, J., Peeters, P.: Reference architecture for holonic manufacturing systems. Computers inIndustry, Vol.37. (1998)

28. Valckenaers, P., Bonneville, F., Van Brussel, H., Wyns, J.: Results of the Holonic Control System Benchmark at KULeuven. In Proceedings of the $4^{\text {th }}$ Rensselaer International Conference on Computer Integrated Manufacturing and Automation Tech., Rensselaer, New York, USA. (1994)

29. Winkler, M., Mey, M.: Holonic Manufacturing Systems. Europe Production Engineering, Vol.18. (1994) 